Pacific Journal of Mathematics

NON-HAUSDORFF CONVERGENCE SPACES 


\title{
NON-HAUSDORFF CONVERGENCE SPACES
}

\author{
R. J. GAZIK
}

In this paper we will establish a method of removing the Hausdorff assumption from certain convergence space theorems. As specific applications the precise form of the closure of a compact set in a regular non-Hausdorff space is given and the exact relationship between $\mathrm{cl}$ and $\mathrm{cl}^{2}$ in a non-Hausdorff compact regular space is obtained. Necessary and sufficient conditions that the transition space for this procedure be topological or pretopological are found and a few embedding theorems are obtained.

1. Introduction. Taking a hint from Thompson [6], who used the "Spiral" relation to investigate properties of topological maps with nonHausdorff domains, let us define $(x, y) \in \mathrm{Sp}$ to mean there exists a filter which converges to both $x$ and $y$. Now Sp is an equivalence relation if and only if the base space is transitive in the sense that if $\mathbf{F} \rightarrow x, y$ and $\mathbf{G} \rightarrow$ $y, z$ then there exists a filter $\mathbf{H} \rightarrow x, z$. Since we want to take quotients by Sp our point of view is that only the class of transitive spaces will be considered. This class is quite broad as the next result shows.

Proposition 1. Regular spaces are transitive, spaces induced by uniform convergence structures are transitive, products of transitive spaces are transitive.

Proof. If $\mathbf{F} \rightarrow x, y$ and $\mathbf{G} \rightarrow y, z$ then $\operatorname{cl} \mathbf{F}<\dot{y}, \operatorname{cl} \mathbf{G}<\dot{y}$ so $\operatorname{cl} \mathbf{F} \vee \operatorname{cl} \mathbf{G}$ exists and converges to $x, z$ by regularity.

If the convergence is uniformizable [1] we may assume there is a base of symmetric filters each of which is coarser than the diagonal filter. Thus, if $\mathbf{F} \rightarrow x, y$ and $\mathbf{G} \rightarrow y, z$ there is some member $\Phi$ of the base such that each of the filters $\mathbf{F} \times \dot{x}, \mathbf{F} \times \dot{y}, \mathbf{G} \times \dot{z}$ is finer than $\Phi$. So if $V \in \Phi$ then $V(x) \cap V(y) \neq \varnothing$ and $V(y) \cap V(z) \neq \varnothing$. A computation now shows $(x, z) \in V^{4}$ hence $\Phi^{4}(x) \rightarrow x$, and the space is transitive.

If filters converge, in the product, to $f, g$ and $g, h$ then use transitivity in each component $X(\lambda)$ to obtain filters which converge to $f(\lambda), h(\lambda)$. Then the product filter converges to $f, h$. This ends the proof. 
A characterization of transitive spaces or a general method of removing the Hausdorff assumption from theorems which involve them seems to be difficult to obtain. In $\$ 2$ a small subclass of the transitive spaces is dealt with-namely the regular spaces. In this class we will obtain a method of trading off regularity for Hausdorffness or, in the case where regularity is part of the hypotheses, we will be able to remove the Hausdorff assumption altogether.

Since the space $X / \mathrm{Sp}$ is the transition space for this procedure, it is of interest to know when it is pretopological or topological. Filter conditions on a regular pretopological (topological) $X$ which are necessary and sufficient that $X / \mathrm{Sp}$ be pretopological (topological) are given in $\S 3$. Some embedding properties are also in this section.

\section{Basic result.}

Proposition 2. If $X$ is regular, $\operatorname{Sp}(x)$ is compact for each $x \in X$.

Proof. Notice first that, when $X$ is regular and $\mathbf{F} \rightarrow x, t$ then $\dot{x} \geq \operatorname{cl} \mathbf{F}$ $\rightarrow t$. (Then also $\dot{t} \rightarrow x$.) Hence, in this case, $\operatorname{Sp}(x)$ consists of all $t$ such that $\dot{x} \rightarrow t$. Now if $\mathbf{U}$ is an ultrafilter on $\operatorname{Sp}(x)$ and $U \in \mathbf{U}$ there exists $x(U) \in U$ such that $\dot{x} \rightarrow x(U)$. Then $U \cap \operatorname{cl}\{x\} \neq \varnothing$ for each $u \in \mathbf{U}$ thus $\mathbf{U}=\mathbf{U} \vee \operatorname{cl} \dot{x} \rightarrow x$ and $\operatorname{Sp}(x)$ is compact.

Proposition 3. If $X$ is regular, $\mathrm{Sp}$ is closed in $X \times X$.

Proof. If $\mathbf{A}$ is a filter which converges to $(x, y)$ with $\mathrm{Sp} \in A$, then for each $A \in \mathbf{A}$ there are points $(x(A), y(A)) \in A$ with $\overline{x(A)} \rightarrow y(A)$. (See the proof of Proposition 2.) Let $S(x(A)), S(y(A))$ be the section filters of the nets $x(A), y(A)$ respectively. Notice that if $S$ is a final section of $x(A)$ and $T$ is the corresponding final section of $y(A)$, then $S \subset \operatorname{cl} T$ because $\overline{y(A)} \rightarrow y(A)$. Thus $\dot{x} \geq \mathrm{cl} S(x(A)) \geq \mathrm{cl}^{2} S(y(A)) \rightarrow y$ since the section filters converge to $x, y$ and $X$ is regular. So $\dot{x} \rightarrow y$ and $(x, y) \in \mathrm{Sp}$. This completes the proof.

In what follows $\pi: X \rightarrow X / \mathrm{Sp}$ is the canonical map and $X / \mathrm{Sp}$ carries the quotient structure [2]. The symbol $[x]$ is the equivalence class $\operatorname{Sp}(x)$. 
Proposition 4. If $p$ is a regular convergence structure on $X$ and $q$ is the quotient structure on $X / \mathrm{Sp}$ then

(1) for $A \subset X / \mathrm{Sp}, \mathrm{cl}_{q} A=\pi \mathrm{cl}_{p} \pi^{-1} A$,

(2) $q$ is Hausdorff and regular,

(3) $\mathrm{Sp}$ is the smallest equivalence relation $r$ on $X$ for which $X / r$ is Hausdorff.

Proof. For part (1) let $[x] \in \pi \mathrm{cl} \pi^{-1} A$ so that $[x]=[t], t \in \mathrm{cl} \pi^{-1} A$. Then $\pi^{-1} A \in \mathbf{U} \rightarrow t$ for some ultrafilter $\mathbf{U}$. Hence $A=\pi \pi^{-1} A \in \pi \mathbf{U} \rightarrow t$ so $[t] \in[x] \in \mathrm{cl}_{q} A$. This means that $\pi \mathrm{cl} \pi^{-1} A \subset \mathrm{cl} A$. For the reverse inequality let $\mathbf{A} \rightarrow[x], A \in \mathbf{A}$, so that, according to the definition of $q$, $\mathbf{A} \geq \pi \mathbf{F}$ for some $\mathbf{F} \rightarrow t \in[x]$. Now $\pi^{-1} A \in \pi^{-1} \mathbf{A} \geq \pi^{-1} \pi \mathbf{F} \geq \mathrm{cl} \mathbf{F} \rightarrow t$ so $t \in \mathrm{cl}_{p} \pi^{-1} A$. This completes the proof of (1).

The proof of (2) is from Proposition 1.1 of [4], Proposition 3 and part (1).

For part (3) if $r$ is any equivalence relation on $X$ which makes $X / r$ Hausdorff consider $(x, y) \in \mathrm{Sp}$. Then $\mathbf{F} \rightarrow x, y$ so, since $X / r$ is Hausdorff and the canonical map is continuous, the equivalence classes determined by $x, y$ are equal. Thus $(x, y) \in r$ or $\mathrm{Sp} \in r$ and the proof is finished.

Let us now give a few specific examples of how to remove the Hausdorff assumption from certain theorems by means of the quotient space $X /$ Sp. Define, for $B \subset X, B^{*}=\{x \mid \dot{b} \rightarrow x$ for some $b \in B\}$. It is well known that a compact, regular, Hausdorff space has an idempotent closure operator. The non-Hausdorff form of this is

Proposition 5. If $X$ is a compact regular space and $B \subset X$ then $\mathrm{cl}^{2} B=(\mathrm{cl} B)^{*}$.

Proof. $X / \mathrm{Sp}$ is compact, regular and Hausdorff by Proposition 4 so $\mathrm{cl}_{q}^{2} \pi B=\mathrm{cl}_{q} \pi B$. Then using Proposition 4 and the continuity of $\pi$, $\pi \mathrm{cl}^{2} B \subset \mathrm{cl}_{q}^{2} \pi B=\mathrm{cl}_{q} \pi B=\pi \mathrm{cl} \pi^{-1} \pi B=\pi \mathrm{cl} B^{*}$. However, $\mathrm{cl} B^{*} \subset \mathrm{cl} B$ for if $x \in \mathrm{cl} B^{*}$ there is an ultrafilter $\mathbf{U} \rightarrow x, B^{*} \in \mathbf{U}$ and then there are points $b(U) \in B, x(U) \in U$ with $\overline{b(U)} \rightarrow x(U)$ for all $U \in \mathbf{U}$. So, as in the proof of Proposition 3, $S(b(U)) \geq \operatorname{cl} S(x(U)) \rightarrow x$ hence $x \in \operatorname{cl} B$ because any ultrafilter finer than $S(b(U))$ contains $B$. Then, also, cl $B^{*}=$ cl $B$ for $B \subset B^{*}$. Combining this with $\pi \mathrm{cl}^{2} B \subset \pi \mathrm{cl} B^{*}$ we get $\pi \mathrm{cl}^{2} B=$ $\pi \mathrm{cl} B$. 
Now, assuming the result of the last paragraph let $x \in \mathrm{cl}^{2} B$. Then $\pi(x)=\pi(t), t \in \mathrm{cl} B$, so $t \rightarrow x, t \in \operatorname{cl} B$ or $x \in(\mathrm{cl} B)^{*}$ hence $\mathrm{cl}^{2} B C$ $(\operatorname{cl} B)^{*}$. The reverse inequality is clear since $B^{*} \subset \operatorname{cl} B$ and the proof is finished.

REMARK. The reader should note that, on a compact regular space, $\mathrm{cl}^{4} B=\mathrm{cl}^{2} B$. (See [3].) Moreover, by Proposition 3.1 of [3] and the result above, $\omega X$ closure is given by the convergence in $X$.

Next, it is known that a compact subset of a Hausdorff space is closed. Interchanging the Hausdorff and regular properties we get

Proposition 6. If $X$ is regular and $B \subset X, B$ compact, then $\mathrm{cl} B=B^{*}$. Moreover, the latter set is closed.

Proof. $X / \mathrm{Sp}$ is Hausdorff and $\pi B$ is compact so $\mathrm{cl}_{q} \pi B=\pi B$. By Proposition $4 \pi \mathrm{cl} \pi^{-1} \pi B=\pi B$ so $\mathrm{cl} B \subset \pi \mathrm{cl} \pi^{-1} \pi B=\pi B$ and $\pi \mathrm{cl} B=\pi B$ follows from this. The result now follows as in Proposition 5 and $B^{*}$ is closed for, again as in Proposition 5, $\mathrm{cl} B^{*}=\mathrm{cl} B=B^{*}$. This completes the proof.

It is known that if $f, g$ are continuous functions with Hausdorff range, then the set on which they agree is closed.

Proposition 7. If $f, g: X \rightarrow Y$ are continuous and $Y$ is regular then $\{x \mid \overline{f(x)} \rightarrow g(x)\}$ is closed.

Proof. Apply the known result to $f^{*}, g^{*}: X \rightarrow Y /$ Sp defined by $f^{*}(x)=\pi f(x), g^{*}(x)=\pi g(x)$, noticing that they are well defined and continuous.

3. When $X / \mathrm{Sp}$ is pretopological (topological). The reader is referred to [5] for information on diagonal theorems and their relation to topologies and to [2] and [4] for a general discussion of quotient spaces.

In this section we shall investigate conditions on $X$ such that $X / \mathrm{Sp}$ is pretopological (topological). For a pretopological (topological) regular $X$ necessary and sufficient conditions are found.

In $X /$ Sp define $\mathbf{A} \rightarrow[x]$ if and only if $\pi^{-1} \mathbf{A} \rightarrow y$ for some $y \in[x]$. 
Notice that this convergence, call it $q^{*}$, is not even a convergence structure in general. But in case $X$ is regular $q^{*}$ is a convergence structure which is actually the quotient structure $q$. This fact will enable us to obtain the results of this section.

Proposition 8. If $X$ is regular then $q^{*}$ is a convergence structure and $q^{*}=q$.

Proof. If $p=[x]$ then $\pi^{-1}(\dot{p})=\operatorname{cl} \dot{x} \rightarrow x$ so $\dot{p} \rightarrow p$ relative to $q^{*}$.

If $\mathbf{A} \rightarrow p$ relative to $q^{*}$ then there exists $y$ in $[x]$ such that $\pi^{-1} \mathbf{A} \rightarrow y$ in $X$. Then

$$
\pi^{-1}(\mathbf{A} \wedge \dot{p}) \geq \pi^{-1} \mathbf{A} \wedge \pi^{-1}(\dot{p}) \geq \pi^{-1} \mathbf{A} \wedge \operatorname{cl} \dot{y} \geq \operatorname{cl}\left(\pi^{-1} \mathbf{A} \wedge \dot{y}\right) .
$$

Since $\pi^{-1} \mathbf{A} \wedge \dot{\mathbf{y}} \rightarrow y$ and $X$ is regular, $\pi^{-1}(\mathbf{A} \wedge \dot{p}) \rightarrow y$, and so $\mathbf{A} \wedge \dot{p} \rightarrow p$ relative to $q^{*}$.

Thus $q^{*}$ is a convergence structure and, since $A=\pi \pi^{-1} A, q^{*} \geq q$. Suppose $\mathbf{A} q$ converges to $[x]$. Then $\mathbf{A} \geq \pi \mathbf{F}, \mathbf{F} \rightarrow y \in[x]$ so, by regularity, $\pi^{-1} \mathbf{A} \geq \pi^{-1} \pi \mathbf{F} \geq \operatorname{cl} \mathbf{F} \rightarrow y$. Therefore $q^{*}=q$ and the proof is complete.

Proposition 9. If $X$ is regular, then $X / \mathrm{Sp}$ is pseudo-topological if and only if $X$ has the following property: If $\mathbf{F}$ is a filter on $X$ such that each ultrafilter finer than $\mathbf{F}$ converges to an element of $[x]$, then $\mathbf{F}$ converges to some element of $[x]$.

Proof. Suppose that the condition holds and assume that each ultrafilter finer than $\mathbf{A} q$ converges to $p=[x]$. By Proposition $8, \pi^{-1} \mathbf{B}$ converges to some point of $[x]$ for each ultrafilter $\mathbf{B} \geq \mathbf{A}$ and, using the condition above, it is not hard to see that $\pi^{-1} \mathbf{A}$ converges to some point of $[x]$. Then $\mathbf{A} q$ converges to $p$ by another application of Proposition 8 so $X / \mathrm{Sp}$ is pseudo-topological.

For the converse notice that if $X / \mathrm{Sp}$ is pseudo-topological and each ultrafilter finer than $\mathbf{F}$ converges to some point of $[x]$, then each ultrafilter finer than $\pi \mathbf{F}$ converges to the point $[x]$ and then $\pi \mathbf{F} \rightarrow x$. Hence $\mathbf{F} \geq \pi^{-1} \pi \mathbf{F}$ converges to some point of $[x]$ by Proposition 8 . Therefore, the condition holds.

In what follows $x r y$ means $(x, y) \in \mathrm{Sp}$ and $\hat{X^{\wedge}}$ is the quotient space $X /$ Sp. 
Proposition 10. If $X$ is regular then $X^{\hat{N}}$ is pretopological if and only if for each class of filters $\mathbf{F}(b), b \in B$, such that $\mathbf{F}(b) \rightarrow x(b)$ with $x(b) r x$ it follows that $\wedge \mathbf{F}(b) \rightarrow y$ for some yrx.

Proof. Suppose $X^{\wedge}$ is pretopological and consider a class of filters as given in Proposition 10. Then $\pi \mathbf{F}(b) \rightarrow[x]$ for each $b \in B$ so $\pi \wedge \mathbf{F}(b)=$ $\wedge \mathbf{F} \pi \mathbf{F}(b) \rightarrow[x]$. Hence $\wedge \mathbf{F}(b) \geq \pi^{-1} \pi \wedge \mathbf{F}(b) \rightarrow y \in[x]$.

Now assume that the condition holds and consider $\mathbf{A} \rightarrow[x]$. From this $\pi^{-1} \mathbf{A} \rightarrow y(A) \in[x]$ so, applying the condition to the class of all $\pi^{-1} \mathbf{A}$ where $\mathbf{A} \rightarrow x$, we get $\pi^{-1} \wedge(\mathbf{A} \mid \mathbf{A} \rightarrow[x])=\wedge\left(\pi^{-1} \mathbf{A} \rightarrow \mathbf{A} \rightarrow[x]\right) \rightarrow t$ for some $t \in[x]$. Thus $\wedge(\mathbf{A} \mid \mathbf{A} \rightarrow[x]) \rightarrow[x]$ and $X^{\wedge}$ is pretopological.

Below $\mathbf{N}(x)$ is the neighborhood filter at $x$.

Proposition 11. If $X$ is regular and pretopological, then $X^{\wedge}$ is pretopological if and only if for each $x \in X$ there is a $z \in[x]$ such that $\mathbf{N}(y) \geq \mathbf{N}(z)$ for each $y \in[x]$.

Proof. The neighborhood filters $\mathbf{N}(y) \rightarrow y$ for each $y \in[x]$ hence $\mathbf{N}(y) \rightarrow z \in[x]$ by the previous proposition so, if $\hat{X}$ is pretopological, $\mathbf{N}(y) \geq N(z)$ for some $z \in[x]$. The conclusion follows from this.

Conversely, if the condition holds, consider any class $\mathbf{F}(b)$ of filters with $\mathbf{F}(b) \rightarrow x(b), x(b) r x$. Then $\mathbf{F}(b) \geq \mathbf{N}(x(b)) \geq \mathbf{N}(z)$ for some fixed $z \in[x]$. Thus $\wedge \mathbf{F}(b) \geq N(z)$ and $X^{\wedge}$ is pretopological by Proposition 10 .

Proposition 12. If $X$ is a regular pretopology then a necessary and sufficient condition that $\hat{X}$ be a topology is that (1) and (2) hold:

(1) If $\eta(y) \rightarrow y$ for each $y \in X$ and $\mathbf{F} \rightarrow x$, then there is some t, trx, such that $\vee \wedge \wedge(\eta(z) \mid z r y, y \in F, F \in \mathbf{F}) \rightarrow t$.

(2) Whenever filters $\mathbf{F}(b) \rightarrow x(b), x(b) r x$, then there is some trx such that $\mathbf{F}(b) \rightarrow t$.

Proof. Suppose (1) and (2) hold. Then $\hat{X^{\hat{N}}}$ is pretopological by Proposition 11. so, by Theorem 1.4 of [5], $X^{\wedge}$ is topological if and only if it is diagonal. But condition (1) is precisely the diagonal condition in $X^{\wedge}$ translated back to $X$ using Proposition 8 .

Conversely if $X^{\wedge}$ is topological it is pretopological and diagonal and (1), (2) are exactly these conditions in $\hat{X^{\hat{X}}}$ translated back to $X$.

In [3] a space is said to be symmetric if it is regular and if whenever $\mathbf{F} \rightarrow x$ and $\dot{x} \rightarrow y$ then $\mathbf{F} \rightarrow y$. It is then proved that a convergence space is completely regular if and only if it has a symmetric compactification. 
In the current case note that if $X$ is pretopological and $z \in[x]$ (so that $\dot{z} \rightarrow x)$ then, since $\mathbf{N}(z) \rightarrow z, \mathbf{N}(z) \geq \mathbf{N}(x)$. Thus $X^{\wedge}$ is pretopological by Proposition 11. We have proved

Proposition 13. If $X$ is pretopological and symmetric then $X / \mathrm{Sp}$ is pretopological.

With respect to the next result the reader should note that a regular topological space is already symmetric.

Proposition 14. If $X$ is a regular topological space, then $X / \mathrm{Sp}$ is topological.

Proof. By using Proposition 8 we see from Definition 2.1 of [4] that $\pi$ is an open map. For if $\mathbf{A} \rightarrow[x], \mathbf{A}$ an ultrafilter, then $\pi^{-1} \mathbf{A}$ converges to a point of $[x]$ and maps on $\mathbf{A}$. The if $y \in[x]$ symmetry shows $\pi^{-1} \mathbf{A} \rightarrow y$. Then $X / \mathrm{Sp}$ is topological by Corollary 2.6 of [4].

Proposition 15. If $X$ is regular, $Y$ is $T_{1}$ and $f: X \rightarrow Y$ has these properties:

(1) $f(\mathbf{F}) \rightarrow f(x)$ if and only if $\mathbf{F} \rightarrow x$

(2) $f(x)=f(y)$ implies $\dot{x} \rightarrow y$.

Then $X / \mathrm{Sp}$ is homeomorphic to the range of $f$.

Proof. Define $g: X / \mathrm{Sp} \rightarrow y$ by $g \pi=f$. Then $g$ is well defined by the continuity of $f$; it is $1-1$ by (2). Then property (1) makes $g$ a homeomorphism.

PROPOSITION 16. If $X, Y$ are symmetric and $\mathrm{Sp}_{1}, \mathrm{Sp}_{2}, \mathrm{Sp}_{3}$ are spirals in $X, Y, X \times Y$ respectively then $X / \mathrm{Sp}_{1} \times Y / \mathrm{Sp}_{2}=X \times Y / \mathrm{Sp}_{3}$.

Proof. The mapping $F$ defined by $F(x, y)=\left(\pi_{1}(x), \pi_{2}(y)\right)$, where $\pi_{1}$, $\pi_{2}$, are the natural maps satisfies all conditions of Proposition 15. For the only non-obvious part of this consider $F(\mathbf{G}) \rightarrow F(x, y)$. Then $p_{1} F(\mathbf{G}) \rightarrow$ $\pi_{1}(x), p_{2} F(\mathbf{G}) \rightarrow \pi_{2}(x)$ hence $p_{1} F(\mathbf{G})=\pi_{1}(x)$. This means $\pi_{1} p_{1} F(\mathbf{G}) \geq$ $\pi_{1}(\mathbf{H}), \mathbf{H} \rightarrow t \in \pi_{1}(x)$. By regularity, $p_{1} F(\mathbf{G}) \geq \pi_{1}^{-1} \pi_{1} p_{1} F(\mathbf{G}) \geq \eta_{1}^{-1} \pi_{1}(\mathbf{H}) \geq$ $\mathrm{cl} \mathbf{H} \rightarrow t$. Using symmetry $p_{1} F(\mathbf{G}) \rightarrow x$ and a similar argument shows $p_{2} F(\mathbf{G}) \rightarrow y$ so $\mathbf{C} \rightarrow(x, y)$.

A space $X$ is $\omega$-regular if $\operatorname{cl}_{\omega X} \mathbf{F} \rightarrow x$ whenever $\mathbf{F} \rightarrow x$. Here $\mathrm{cl}_{\omega X} A$ means the closure of $A$ in the finest completely regular topology coarser 
than $X$. Spaces which are $\omega$-regular are of much interest in various questions involving embeddings. See [3] and [7] and their references.

Proposition 17. If $X$ is $\omega$-regular so is $X / \mathrm{Sp}$.

Proof. By Theorem 3.2 of [3] it is sufficient to show that $X / \mathrm{Sp}$ has a regular compactification. But another application of Proposition 15 proves that $R(X) / \mathrm{Sp}$ is a regular compactification of $X / \mathrm{Sp}$ whenever $R(X)$ is a regular compactification of $X$.

For background information on $c$-embedded spaces see [7].

Proposition 18. If $X$ is $\omega$-regular, symmetric and pseudo-topological then $X / \mathrm{Sp}$ is c-embedded.

Proof. This follows from Proposition 17 and the fact that, whenever $X$ is symmetric and pseudo-topological so is $X / \mathrm{Sp}$ by Proposition 9 .

\section{REFERENCES}

[1] C. H. Cook and H. R. Fischer, Uniform convergence structures, Math. Ann., 173 (1967), 290-306.

[2] D. C. Kent, Convergence quotient maps, Fundamenta-Mathematica, 6E (1969), 197205.

[3] D. C. Kent and G. D. Richardson, Completely Regular and w-regular Spaces, Proc. Conf. on Convergence Spaces, Cameron University, April 1980.

[4] __ Open and proper maps between convergence spaces, Czech. J., 23 (1973), 15-23.

[5] Eva Lowen-Colebunders, An internal and external characterization of convergences in which adherences of filters are closed, Proc. Amer. Math. Soc., 72 (1978), 205-209.

[6] Travis Thompson, Concerning certain subsets of non-Hausdorff topological spaces, Bulletino U.M.I. (5) 14-A (1977), 34-37.

[7] G. D. Richardson and D. C. Kent, $A$ note on $C(X)$, Proc. Amer. Math. Soc., 49 (1975), 441-445.

Received July 1, 1981 and in revised form October 2, 1981. The author expresses sincere thanks to the referee for help given in matters of exposition and, also for suggesting revisions in Section 3 which strengthen the paper. Research supported by Epscon NSF grant.

Arkansas State University

STATE UNIVERSITY, AR 72468 


\title{
PACIFIC JOURNAL OF MATHEMATICS \\ EDITORS
}

\author{
Donald BABBITT (Managing Editor) \\ University of California \\ Los Angeles, CA 90024 \\ Hugo Rossi \\ University of Utah \\ Salt Lake City, UT 84112 \\ C. C. MOore and ARTHur OGus \\ University of California \\ Berkeley, CA 94720
}

\author{
J. Dugundi \\ Department of Mathematics \\ University of Southern California \\ Los Angeles, CA 90089-1113
}

R. FINN and H. SAMELSON

Stanford University

Stanford, CA 94305

ASSOCIATE EDITORS
R. ARENS
E. F. BECKENBACH
B. H. NeUmanN
F. WOLF
K. YosHidA
(1906-1982)

\section{SUPPORTING INSTITUTIONS}

\author{
UNIVERSITY OF ARIZONA \\ UNIVERSITY OF BRITISH COLUMBIA \\ CALIFORNIA INSTITUTE OF TECHNOLOGY \\ UNIVERSITY OF CALIFORNIA \\ MONTANA STATE UNIVERSITY \\ UNIVERSITY OF NEVADA, RENO \\ NEW MEXICO STATE UNIVERSITY \\ OREGON STATE UNIVERSITY
}

\author{
UNIVERSITY OF OREGON \\ UNIVERSITY OF SOUTHERN CALIFORNIA \\ STANFORD UNIVERSITY \\ UNIVERSITY OF HAWAII \\ UNIVERSITY OF TOKYO \\ UNIVERSITY OF UTAH \\ WASHINGTON STATE UNIVERSITY \\ UNIVERSITY OF WASHINGTON
}

The Supporting Institutions listed above contribute to the cost of publication of this Journal, but they are not owners or publishers and have no responsibility for its content or policies.

Mathematical papers intended for publication in the Pacific Journal of Mathematics should be in typed form or offset-reproduced (not dittoed), double spaced with large margins. Please do not use built up fractions in the text of the manuscript. However, you may use them in the displayed equations. Underline Greek letters in red, German in green, and script in blue. The first paragraph must be capable of being used separately as a synopsis of the entire paper. In particular it should contain no bibliographic references. Please propose a heading for the odd numbered pages of less than 35 characters. Manuscripts, in triplicate, may be sent to any one of the editors. Please classify according to the scheme of Math. Reviews, Index to Vol. 39. Supply name and address of author to whom proofs should be sent. All other communications should be addressed to the managing editor, or Elaine Barth, University of California, Los Angeles, California 90024.

There are page-charges associated with articles appearing in the Pacific Journal of Mathematics. These charges are expected to be paid by the author's University, Government Agency or Company. If the author or authors do not have access to such Institutional support these charges are waived. Single authors will receive 50 free reprints; joint authors will receive a total of 100 free reprints. Additional copies may be obtained at cost in multiples of 50 .

The Pacific Journal of Mathematics is issued monthly as of January 1966. Regular subscription rate: $\$ 132.00$ a year (6 Vol., 12 issues). Special rate: $\$ 66.00$ a year to individual members of supporting institutions.

Subscriptions, orders for numbers issued in the last three calendar years, and changes of address should be sent to Pacific Journal of Mathematics, P.O. Box 969, Carmel Valley, CA 93924, U.S.A. Old back numbers obtainable from Kraus Periodicals Co., Route 100, Millwood, NY 10546.

The Pacific Journal of Mathematics ISSN 0030-8730 is published monthly by the Pacific Journal of Mathematics at P.O. Box 969, Carmel Valley, CA 93924. Application to mail at Second-class postage rates is pending at Carmel Valley, California, and additional mailing offices. Postmaster: Send address changes to Pacific Journal of Mathematics, P. O. Box 969, Carmel Valley, CA 93924.

PUBLISHED BY PACIFIC JOURNAL OF MATHEMATICS, A NON-PROFIT CORPORATION

Copyright $(1983$ by Pacific Journal of Mathematics 


\section{Pacific Journal of Mathematics}

Vol. 106, No. 2 December, 1983

R. J. Gazik, Non-Hausdorff convergence spaces $\ldots \ldots \ldots \ldots \ldots \ldots \ldots 257$

John Norman Ginsburg, A note on the cardinality of infinite partially

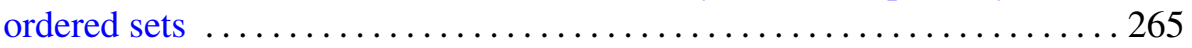

Norman Joseph Goldstein, Ampleness in complex homogeneous spaces

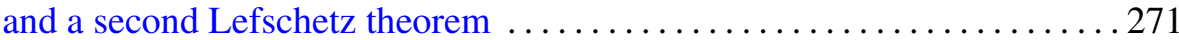

Clyde D. Hardin and Loren Dallas Pitt, Integral invariants of functions and

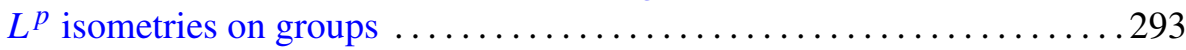

M. Scott Osborne and Garth William Warner, Jr., The Selberg trace formula. II. Partition, reduction, truncation 307 